\title{
A RANDOMISED CONTROLLED STUDY TO EVALUATE THE EFFEC- TIVENESS OF SHORTWAVE DIATHERMY IN ACUTE SINUSITIS
}

\section{Anand B Heggannavar*1, Anil S Harugop², Divya M Madhale ${ }^{3}$, Linata S Walavalkar .}

${ }^{* 1}$ Associate P rofessor, Orthopaedic Manual Therapy, KLE University, Institute of Physiotherapy, Belagavi, Karnataka, India.

${ }^{2}$ Head of the Department and Professor, E.N.T and H.N.S, Jawaharlal Nehru Medical College, Belagavi, Karnataka, India.

${ }^{3,4}$ Physiotherapist, KLE University. Institute of Physiotherapy, Belagavi, Karnataka, India.

\section{ABSTRACT}

Background: Sinusitis is the inflammation of the mucous lining of any or all of the paranasal sinuses. It is a very common condition with a high incidence rate. It also shows high recurrence of symptoms which causes high damage to the patient and disables him/her to carry out daily activities efficiently. Availability of medical options has increased but so has the rate of antibiotics resistance. Therefore, it becomes a necessity to prevent recurrence of symptoms by providing an adequate overall treatment in the initial i.e. the acute phase of sinusitis. Shortwave diathermy is a deep heating modality. Extensive literature is available on various medical and surgical treatment options for sinusitis but there is lack of sufficient evidence on use of shortwave diathermy- a physiotherapy modality, for the cases of sinusitis. Hence, this study is done to evaluate the effectiveness of shortwave diathermy in acute sinusitis.

Materials and Methods: 30 subjects between 18- 50 years age with acute sinusitis were referred by E.N.T. department of KLES Dr. Prabhakar Kore Hospital. They were divided into 2 groups with 15 subjects in each group randomly where allocation was done by envelope method. Subject in Group A were treated with placebo SWD (20 minutes) and medications for 5 days. Subjects in Group B were treated with shortwave diathermy (20 minutes) and medications for 5 days. Outcome was measured with help of SNOT-22 (Sinonasal Outcome Test -22) questionnaire which was filled on first day prior to the treatment session and on the fifth day after the completion of five treatment sessions. To assess the changes in pre-treatment and post-treatment symptoms in both the groups, the data was analyzed with the paired t-test.

Results: The percentage of change in SNOT-22 scores pre-treatment and post-treatment in Group A was $23.36 \%$ whereas in Group B was $46.54 \%$.

Conclusion: Shortwave diathermy along with medications is more effective then placebo SWD along with medications in treatment of acute sinusitis.

KEY WORDS: Acute sinusitis, Shortwave Diathermy, Sinonasal Outcome Test-22.

Address for correspondence: Dr Anand B Heggannavar, MPT, Associate Professor, Orthopaedic Manual Therapy, KLE University, Institute of Physiotherapy, Belagavi, Karnataka, India.

E-Mail: anandhegs@yahoo.co.in

\begin{tabular}{|c|c|c|}
\hline \multicolumn{3}{|c|}{ Access this Article online } \\
\hline $\begin{array}{l}\text { Quick Response code } \\
\qquad \text { 口is }\end{array}$ & \multicolumn{2}{|c|}{$\begin{array}{l}\text { International Journal of Physiotherapy and Research } \\
\text { ISSN 2321- } 1822 \\
\text { www.ijmhr.org/ijpr.html }\end{array}$} \\
\hline DoI: $10.16965 /$ ijpr.2017.137 & $\begin{array}{l}\text { Received: 16-03-2017 } \\
\text { Peer Review: 17-03-2017 } \\
\text { Revised: None }\end{array}$ & $\begin{array}{l}\text { Accepted: } 24-04-2017 \\
\text { Published (O): 11-06-2017 } \\
\text { Published (P): 11-06-2017 }\end{array}$ \\
\hline
\end{tabular}

\section{INTRODUCTION}

The sinuses (also known as the paranasal sinuses) viz. frontal, maxillary, sphenoidal and ethmoidal are air filled spaces present within some bones around the nasal cavities [1]. They open into the nasal cavity through its lateral wall. 
Sinusitis is the inflammation of the mucous lining of any or all paranasal sinuses [2]. It has an incidence rate of about more than 10 million cases per year in India [3] and there by forms a huge disease burden on the otorhinolaryngologists.

In an article in Times of India, it was reported that an estimated 134 million Indians i.e. 1 in 8 Indians, suffer from chronic rhinosinusitis, the symptoms of which include are not limited to debilitating headaches, fever and nasal congestion obstruction. This estimate by The National Institute of Allergy and Infection Diseases (NIAID) is exclusive of the suffering from acute sinusitis [4].

In a study done by Sahlstrand-Johnson $\mathrm{P}$, Ohlsson $B$, Von Buchwald C, Jannert M, AhIner-Elmquist $M$, on quality of life and absenteeism in patients with chronic rhinosinusitis concluded that, twenty-eight percent of the patients had probable anxiety or depression disorders and fifty seven percent of the patients reported absenteeism from work due to sinus problems [5].

Sinusitis can be classified into four types namely, acute (2-4 weeks), sub-acute (4-8weeks), chronic (>8weeks) and recurrent sinusitis (occurs more than thrice a year).

The maxillary sinuses are the most frequently infected sinuses due to its close proximity to teeth and due to inadequate drainage because of the higher level of the ostium. The frontal and the ethmoidal sinuses are less frequently affected whereas sphenoidal sinuses are seldom infected. Nasal infections, dental infections, fungal infections, trauma, blood-borne infections, neighboring infections (tonsillitis), lowered resistance, chill and atmospheric pollution form the predisposing factors to sinusitis [6].

The causative organisms for sinusitis may be viruses or bacteriae. Usually the infection is caused by the upper respiratory tract organisms like Streptococci, Pneumococci, Micrococcus catarrhalis or Haemophilus influenza. However, gram negative bacteria like Bacillus pyocyaneus, Bacillus coli and Bacillus necrodentalis are dominant, if the infection spreads from an infected tooth.

Various symptoms such as pain, nasal discharge which may or may not be foul smelling, nasal resonance, dry cough, epistaxis, malaise, headache and fever may be observed.

Treatment of sinusitis is mainly medical and includes decongestants, antibiotics, nasal sprays etc.[7] In extreme cases i.e. in cases with troublesome recurrent sinusitis, surgical options are balloon sinuplasty and FESS (Functional Endoscopic Sinus Surgery) are tried.

High recurrence rates are seen despite of extensive use of medications due to development of resistance to medicines. Repeated occurrence of symptoms cause high damage to the patient and disables him/her to carry out daily activities efficiently.

The cost of available treatment options increases the financial burden on the patient as well as on the country's financial resource. Various studies are done across the world to estimate the annual cost for the treatment of sinusitis, one of which [8] reports that, chronic sinusitis cases cause the maximum expenditure as compared to the other types of sinusitis. Therefore it becomes necessary to control the symptoms and prevent development of resistance to medications at the earliest stage of sinusitis so to prevent further damage and also to control recurrence rates.

Physiotherapy treatment for sinusitis includes the application of shortwave diathermy to the affected sinuses. Shortwave diathermy is a non-ionizing radiation from the radio frequency portion of the electromagnetic spectrum. Reference to the medical use of high-frequency electrical current, dates back to 1890s, when d'Arsonval passed a 1 ampere current at high frequency through himself and his assistant [9]. A shortwave diathermy current frequency of between $10^{7}$ and $10^{8} \mathrm{~Hz}$ and sets up radio waves with a wavelength of between 30 and $3 \mathrm{~m}$, but the diathermy current commonly used for medical purpose has a frequency of $27.12 \mathrm{MHz}$ and sets up radio waves with a wavelength of $11 \mathrm{~m}[10]$.

Shortwave diathermy is a deep heating modality. Its principle effect on the body tissues is heat production and other effects result from the increase in temperature. Increase in temperature leads to vasodilation of the blood vessels 
thereby increasing the blood supply to the damaged tissue, which also increases white blood cells, antibodies other nutritive materials which further help in the minimization or resolution of the inflammation [11].

Pain relief is another effect of shortwave diathermy, exact mechanism remains unknown but it is assumed that pain relief occurs due to the sedative effect of shortwave diathermy on sensory nerves, counter-irritation by heat, resolution in inflammation and there relief of underlying muscle spasm [11].

Evaluation of symptoms of sinusitis and quality of life can be done using various tests, some of which include, the short form 36-item questionnaire (SF-36), the Hospital Anxiety and Depression Scale(HAD), SNOT-22 (Sino-nasal Outcome Test), and Visual Analogue Scale.

Availability of various medical options has increased but so has the rate of resistance. Limitations are faced in the treatment of sinusitis due to lack of sufficient evidence on the other treatment modalities like shortwave diathermy. No recent and advanced literature is available on the effectiveness of shortwave diathermy in acute cases of sinusitis. There by awakening an immediate and strong need for this study to be conducted. Hence this study is focused to evaluate the effectiveness of shortwave diathermy in sinusitis through a randomized controlled trial.

\section{MATERIALS AND METHODS}

30 subjects suffering with acute sinusitis were referred for the study by the E.N.T department of KLES Dr. Prabhakar Kore Hospital.

The inclusion criteria for the above subjects were; a) Case diagnosed as acute sinusitis. b) All genders. c) Age group- 18-50yrs.

The exclusion criteria were; a) Nasal obstructions: Deviated nasal septum, hypertrophic turbinates, polyps and new growths; any blockage to free drainage of sinus b) Diabetes, Hypertension c) Tuberculosis, Pregnant Women d) Space Occupying Lesions e) Metal implants (dental implants, any other implants) f) Cardiac failure g) Low blood pressure, dizziness.

The study was carried out in KLES. Dr Prabhakar Kore Hospital and Medical Research Centre,
Belagavi. The study was approved by Institutional Ethical Committee of KLEU Institute of Physiotherapy, Belagavi.

Outcome measure: The outcome measure used was SNOT-22 (Sinonasal Outcome Test-22). SNOT- 22 consists of 22 symptomatic problems faced by the subjects. Each question was answered on basis of 6 cut points. 0 indicates no problem and 5 indicates problem as bad as it can be. On the extreme right, subjects were asked to mark 5 most troubling symptoms.

Intervention: The participants who had reported to KLES Dr. Prabhakar Kore Hospital, MRC and were diagnosed by the ENT Department as cases of acute sinusitis were screened for their eligibility and were invited to participate in the study. The purpose of the study was explained and a written informed consent was obtained from all the participants fulfilling the inclusion and exclusion criteria. All the subjects were asked to fill the SNOT-22 prior to the first treatment session. Following this the subjects were randomly allocated using the envelope method to two groups: Group A (control group) received the standard treatment protocol of analgesics, antibiotics and nasal decongestants for a period of 5 days.Tablet Amoxiclav (625mg, 1-01), Tablet Paracetamol (500mg, 1-0-1), Tablet Levocetrizene (5mg, 0-0-1), Xylometazoline Nasal Drops $\left(3^{\circ} / 3^{0} / 3^{0}\right)$ and Steam Inhalation (1-1-1). Along with the medical therapy, these subjects received placebo treatment by shortwave diathermy. The position of the patient and the placement of the electrodes remained same as the Group B (as is described below). And Group B received continuous shortwave diathermy for duration of 20 minutes in each session for 5 days along with the above mentioned medical treatment. A standard cross-fire method was used to treat all the sinuses. Position of the patient: Supine lying Positioning of the electrodes: For the treatment of all sinuses (including ethmoidal), one electrode was placed on the lateral part of the forehead, and the other on the opposite side of the face, below the angle of jaw. After 10 minutes of treatment, the placement of the electrodes was changed to opposite sides, this cause heating of the sinuses equally. Treatment for another 10 minutes was given with electrodes in this position. Intensity 
was set according to the tolerance of the patient and also so as to produce only minimal heating of the sinuses. Every treatment session, subjects were assessed for any sort of inconvenience encountered during or after the treatment. Postcompletion of the treatment duration (i.e. 5 sessions), subjects of both the groups were asked to fill in the SNOT-22 and the differences in the pre-treatment and post-treatment scores were evaluated.

\section{RESULTS}

The results were analyzed by comparing pre-treatment and post-treatment scores of SNOT-22 Questionnaire.

The statistical analysis was done using SPSS software Version 21 for statistical measures such as mean, standard deviation, paired t test and test of significance. Kolmogorov-Smirnov Z test was applied to analyze the SNOT- 22. Comparison of the pre-treatment and post-treatment scores was done using the students paired t test.

Gender Distribution: This study included 30 participants split into Group A and Group B and each comprised of 15 participants. The no. of males and females included in both the groups were 03 and 12 respectively; that forms $20 \%$ of males and $80 \%$ of females in study. (Table 1 and fig. 1 and fig. 2)

Age Distribution: Participants included in this study were 18-50 (mean age 23.6) years of age group. The minimum and the maximum age of the participants included in the study were 19.00 and 50.00 respectively. The minimum and the maximum age of the participants included in Group A were 19.00 and 50.00 respectively and that in the Group B were 19.00 and 25.00 respectively. Mean age of the participants in Group A and Group B was 25.07 and 22.13 respectively. (Table 1 and fig. 1 and 2)

Fig. 1:

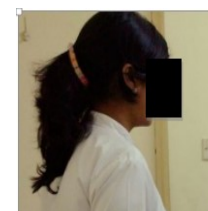

Application of SWD.
Table 1: Distribution of Male and Females and Comparison of Group A and Group B with Mean Age by $t$ Test.

\begin{tabular}{|c|c|c|c|c|c|}
\hline & Male & Female & Mean Age \pm SD & t value & $p$ value \\
\hline Group A & 3 & 12 & $25.07 \pm 8.83$ & \multirow{2}{*}{1.2466} & \multirow{2}{*}{0.2229} \\
\hline Group B & 3 & 12 & $22.13 \pm 2.26$ & & \\
\hline
\end{tabular}

Fig. 2: Application of SWD

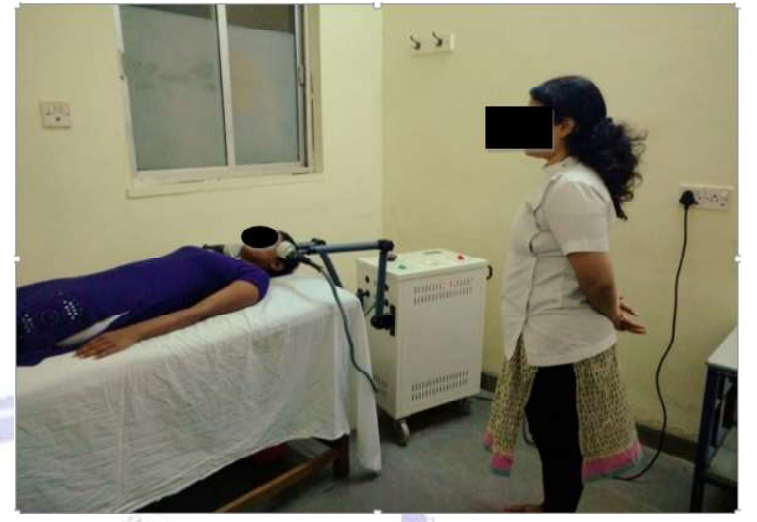

SNOT-22 Questionnaire: In Group A, the mean pre-test score was 46.80 and the post-test score was 35.87. Mean difference between pre-test and post- test was 10.93 . There was $23.36 \%$ of change in symptoms and the t-value was 6.6542 and $p$ value was 0.0001

In Group B, the mean pre-test score was 46.27 and the post-test was 24.73. Mean difference between pre-test and post-test was 21.53 . There was $46.54 \%$ of change in symptoms and t-value was 11.3661 and $p$ value was 0.0001 . (Table 2 and fig. 3)

Table 2: With in Comparison of Sino nasal outcome test scores in group A and group B by dependent $t$ test.

\begin{tabular}{|c|c|c|c|c|c|c|}
\hline Group & Time & Mean $\pm S D$ & Mean Diff. & $\begin{array}{c}\% \text { of } \\
\text { change }\end{array}$ & Paired t & P-value \\
\hline \multirow{2}{*}{ Group A } & Pretest & $46.80 \pm 13.75$ & \multirow{2}{*}{10.93} & \multirow{2}{*}{23.36} & \multirow{2}{*}{6.6542} & \multirow{2}{*}{$0.0001^{*}$} \\
\hline & Posttest & $35.87 \pm 10.33$ & & & & \\
\hline \multirow{2}{*}{ Group B } & Pretest & $46.27 \pm 13.16$ & \multirow{2}{*}{21.53} & \multirow{2}{*}{46.54} & \multirow{2}{*}{11.3661} & \multirow{2}{*}{$0.0001^{*}$} \\
\hline & Posttest & $24.73 \pm 8.98$ & & & & \\
\hline
\end{tabular}

$*_{\mathrm{p}}<0.05$

Fig. 3: Distribution of respondents in Group A and Group $B$ by age groups.

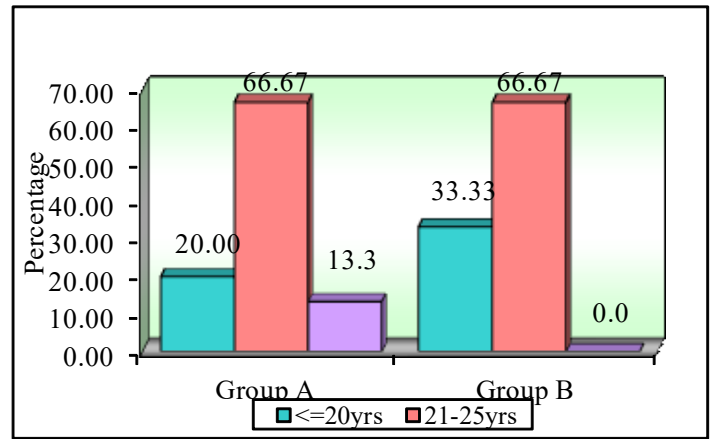


In Group A, mean score on pre-test is $46.80 \pm$ 13.75 while in group $B$ mean score on pre-test is $46.27 \pm 13.16$ with the t-value as 0.1085 and p-value as 0.9143 . In group $A$, the mean score on post-test was $35.87 \pm 10.33$ while in group $B$ the mean score on post-test was $24.73 \pm 8.98$ with the t-value as 3.1504 and $p$ value as 0.0039 . The difference between pre-test and post-test scores in Group A was $10.93 \pm 6.36$ and the difference between pre-test and post-test scores in group $B$ was $21.53 \pm 7.34$ with the $t$-value as 4.2269 and $p$-value as 0.0002 . (Table 3 and fig. 4)

Table 3: Between Group Comparison of group A and group $B$ with Sino nasal outcome test scores by independent $t$ test.

\begin{tabular}{|c|c|c|c|c|}
\hline Variable & Group & Mean \pm SD & t-value & p-value \\
\hline \multirow{2}{*}{ Pretest } & Group A & $46.80 \pm 13.75$ & \multirow{2}{*}{0.1085} & \multirow{2}{*}{0.9143} \\
\cline { 2 - 3 } & Group B & $46.27 \pm 13.16$ & & \\
\hline \multirow{2}{*}{ Posttest } & Group A & $35.87 \pm 10.33$ & \multirow{2}{*}{3.1504} & $0.0039 *$ \\
\cline { 2 - 3 } & Group B & $24.73 \pm 8.98$ & & \\
\hline \multirow{2}{*}{ Difference } & Group A & $10.93 \pm 6.36$ & \multirow{2}{*}{4.2269} & $0.0002 *$ \\
\cline { 2 - 3 } & Group B & $21.53 \pm 7.34$ & & \\
\hline
\end{tabular}

Fig. 4: Comparison of pretest and posttest sinonasal outcome test scores in Group A and Group B.

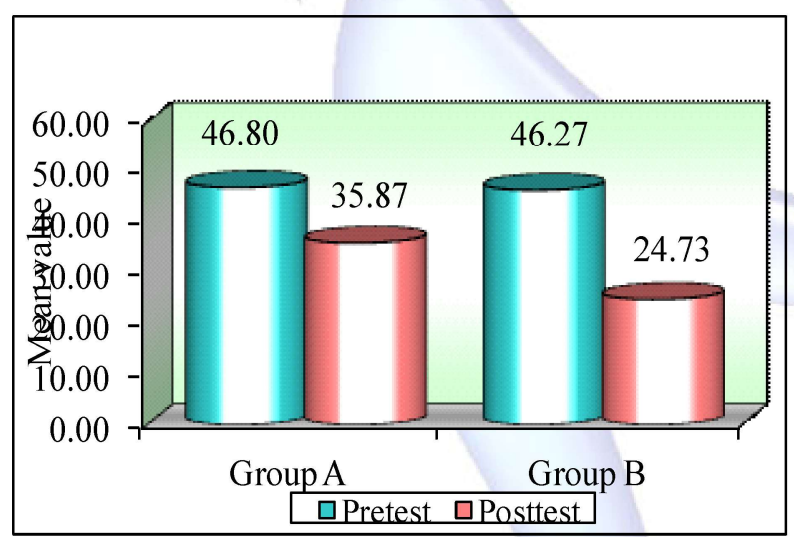

\section{DISCUSSION}

The present study was conducted to evaluate the effectiveness of shortwave diathermy in acute sinusitis. 30 subjects were included in study on basis of inclusion and exclusion criteria. Age group included in the study was 18 to 50 years. In the present study 3 males and 12 females were included in each group in the study. This coincides with the study conducted by Elisabeth $\mathrm{H}$. Ference, Bruce K. Tan and Stephanie Shintani Smith on gender difference in prevalence, treatment and quality of life of patients with chronic rhinosinusitis and concluded that women are more likely reporting with symptoms [12]. With the help of envelope method the subjects were allocated into two groups.

Group A included 15 subjects who were treated with placebo SWD (equipment switched off) for 20 minutes in conjunction with medical treatment for 5 days. According to the study conducted by Sng W J, Wang DY on efficacy and side effects of antibiotics in the treatment of acute rhinosinusitis. It was concluded that usage of antibiotics in ARS is widespread and there seems to be only slight added benefit in the usage of antibiotics over placebo. They also suggested further studies to be conducted for confirm results [13].

Group B included 15 subjects who were treated with shortwave diathermy for 20 minutes along with medical treatment for 5 days. In a study, conducted on shortwave diathermy in treatment of nasal sinusitis inferred that SWD can be used alone or as an adjunct to other measures in the management of inflammation process of the accessory nasal cavities [14].

The outcome measure used was SNOT-22 (Sinonasal Outcome Test-22). SNOT- 22 consists of 22 symptomatic problems faced by the subjects. Each question was answered on basis of 6 cut points. 0 indicates no problem and 5 indicates problem as bad as it can be. On the extreme right subjects are asked to mark 5 most troublesome symptoms. All the subjects were given SNOT-22 Questionnaire to fill after allocation to the study and also at the end of $5^{\text {th }}$ treatment session. Later, pre-treatment and post- treatment scores were analyzed. This coincides with the study by C. Hopkins, S. Gillett, R. Slack, V. J. Lund, J. P. Browne on the psychometric validity of the 22 - item Sinonasal Outcome Test, it was derived that the SNOT-22 is valid and easy to use as it has high internal consistency (The Cronbach's alpha scores for SNOT-22 were 0.91). The test-retest reliability coefficient was 0.93 , indicating high reliability of repeated measures [15]. SNOT -22 Questionnaire had more items in a modified manner included in it in comparison to SNOT 20 and SNOT 18. Therefore, SNOT-22 was included in study. The results from the statistical analysis of the present study suggests that shortwave diathermy along with medications is more 
effective than SHAM treatment with medications in case of acute sinusitis. There was significant improvement in symptoms in all subjects in Group B according to difference in their pre-treatment and pre-treatment scores.

The present study is similar to study conducted by Neesha Shinde and Shinde Kiran Jayawant on efficacy of Shortwave diathermy in patients with sinusitis in each group; Group A was treated with only shortwave diathermy, Group B was tread with shortwave diathermy and medical treatment and group $C$ was treated with medicines alone. They concluded that shortwave diathermy is as beneficial as medications when administrated individually. It also concluded that when shortwave diathermy and medications are used in combination, it was more beneficial with little or no recurrence of sinusitis [16].

Shortwave diathermy's main principle is heating effect. Heat kills the positive organisms like streptococci, pneumococci, etc. producing symptomatic relief. Heat also causes vasodilation that leads to increase in blood flow washing out the nociceptive materials that will reduce pain and increase in blood flow will help in healing of the damaged tissues resulting in reduction in inflammation and also in relieving spasm. Thus, all the above factors in correlation would have been reason for relieving of the symptoms by shortwave diathermy.

The study done by W.P.E. Paterson, M.D. in his study on the treatment of nasal sinus infection by ultra -shortwave diathermy among 126 acute and sub- acute cases treated for sinus conditions over a period of three years. It was interpreted that shortwave diathermy has a positive bactericidal effect on streptococci and it also results in increased phagocytosis resulting in early healing of sinusitis [17].

Geoffrey. C. Goats [PhD, MCSP] in his research on continuous shortwave diathermy (radiofrequency) concluded that pulsed SWD was effective for acute cases while continuous SWD was effective for sub-acute and chronic conditions, he stated that continuous shortwave diathermy can help to relieve pain and reduce muscle spasm and reduce inflammatory states and reduce swelling [18].

In the present study SNOT- 22 Questionnaire was used and there was difference in pre-treatment and post-treatment scores in both groups. But in group $B$ difference was more significant than in Group A. This shows that there was significant reduction in symptoms in subjects in Group B that will help them to carry out their daily activities efficiently. This study concludes that shortwave diathermy along with medications in more effective then SHAM treatment along with medications in case of acute sinusitis.

\section{CONCLUSION}

The present study concludes that shortwave diathermy along with medications is more effective than placebo SWD treatment along with medications.

\section{ACKNOWLEDGEMENTS}

The authors are thankful to KLEU Institute of Physiotherapy and KLES Dr. Prabhakar Kore Hospital and MRC, Belagavi for granting us the opportunity and permission of conducting this study and also for providing the necessities of the study. We are grateful to all our subjects for considering participation in our study and also for giving us their valuable feedback regarding the treatment.

\section{Conflicts of interest: None}

\section{REFERENCES}

[1]. B.D. Chaurasia's Human Anatomy; 5th edition; Volume 3; Page no. 230.

[2]. Fundamentals of Ear, Nose and Throat and HeadNeck Surgery; 9th edition.

[3]. According to statistics (Aug.2016), Sinusitis is a very common condition and accounts for more than 10 million cases per year in India,www.google.co.in/search?q=Sinusitis TOI, Pratibha Masand |TNN |Apr 11,2012,12.14 AM IST.

[4]. Sahlstrand- Johnson P, Ohlsson B, VonBuchwald C, Jannert M, Ahlner-Elmquist M; A multi- center study on quality of life and absenteeism in patient with chronic rhinosinusitis referred for endoscopic surgery.Rhino,2011 Oct;420-8. DOI: 10:4193/ Rhino11.101 PIMD:21991567.

[5]. A Short Textbook of E.N.T. Diseases by K.B. Bhargava, S.K.Bhargava, T.M.Shah; Page no. 192.

[6]. Kerr A, Groves's j, Scott-Brown's otolaryngology. London: Butterworths; 1987.

[7]. Smith K, Orlandi RR, Rudmik L performed a systematic review to estimate the cost of adult chronic rhinosinusitis and it was concluded that overall annual economic burden of CRS in the United States 
estimates to be $\$ 22$ billion 014 USD (direct and indirect costs). Laryngoscope, 2015 Jul; 1547-56. DOI:10.1002/LARY.25180. Epub 2015 Jan 30. PIMD:25640115.

[8]. Electrotherapy : Evidence Based Practice Edited by Sheila Kitchen; 11th edition; Page no. 145.

[9]. Clayton's Electrotherapy: Theory and Practice by Angela Fosster and Nigel Palastanga; 9th edition; Chapter 4; Page no. 112 \& Page no 128.

[10]. Basics of electrotherapy by Subhash M Khatri; 2nd edition; Page no. 75,77,78.

[11]. Ference EH, Tan BK, Hulse KE, Chandra RK, Smith SB, Kern RC, Conley DB, Smith SS. Commentary on gender differences in prevalence, treatment, and quality of life of patients with chronic rhinosinusitis. Allergy \& Rhinology. 2015;6(2):e82.

[12]. Sing W J, Wang DY on efficacy and side effects of antibiotics in the treatment of acute rhinosinusitis. Rhinology. 2015 Mar; 53(1):3-9. Doi: 10.4193/ Rhin13.225.

[13]. Hollender A. shortwave diathermy in treatment of nasal sinusitis and allergic rhinitis. Journal of the American Medical Association. 1942;118(7):507. Available from: http://dx.doi.org/10.1001/ jama.1942.02830070013004.
[14]. Hopkins C, Gillet S, Slack R, Lund V, Browne J. Psychometric validity of the 22-items Sinonasal Outcome Test. Clinical Otolaryngology. 2009;34(5):447454. Available from: http://dx.doi.org/10.1111/ j.1749-4486.2009.0995.x.

[15]. 'Efficacy of Shortwave Diathermy in Patients with Sinusitis' - a study by Neesha Shinde, Shinde Kiran Jayawant. International Journal of Health Science \& Research (www.ijhsr.com)2002;2(4).

[16]. 'The treatment of nasal sinus infection by ultrashortwave' by W.P.E. Paterson. M.D. Ottawa; The Canadian Medical Association Journal [May1940] Read at the Seventh Annual Meeting of the Canadian Medical Association, Section of Otolaryngology, Montreal, June22 1939.

[17]. Goats G. Continuous short-wave radio-frequency) diathermy. British Journal ofSportsMedicine.1989; 23(2):123127. Availablefrom:http://dx.doi.org/ 10.1136/bjsm.23.2.123.

How to cite this article:

Anand B Heggannavar, Anil S Harugop, Divya M Madhale, Linata S Walavalkar. A RANDOMISED CONTROLLED STUDY TO EVALUATE THE EFFECTIVENESS OF SHORTWAVE DIATHERMY IN ACUTE SINUSITIS. Int J Physiother Res 2017;5(3):2066-2072. DOI: 10.16965/ijpr.2017.137 